\title{
La visión de la Procuraduría para la Defensa de los Derechos Humanos y sus necesarias prioridades
}

El Procurador para la Defensa de los Derechos Humanos es una figura moral. Acostumbrados a la presentación de ejemplos de moralidad, ubicados en siglos pasados y demasiado alejados de nosotros, o al culto a personalidades contemporáneas, que con el paso del tiempo se han revelado como deshonestas e incluso claramente criminales, el país necesita instituciones en las que creer. La propia tradición de valorar a los ciudadanos según el éxito económico o político que tengan, alabando incluso las prácticas autoritarias en el poder y el compadrazgo entre poderes y poderosos, ha hecho descender la confianza, tanto en personas como en instituciones. La palabra política es para muchos sinónimo de suciedad y de trampa. El término justicia, de arreglo y contubernio. $Y$ aunque se pueda hablar de síntomas de recomposición social en algunos estratos, lo cierto es que se camina lento, y que abundan los ejemplos negativos. Por ello, muchos de los esfuerzos que se hacen no son suficientes para encauzar el desarrollo cívico ciudadano.

Como diría Roque Dalton, el dolor en nuestra patria es demasiado antiguo, presente en demasiadas personas explotadas, "en el crepúsculo, en el sol calcinante, en la ficticia aurora cotidiana... y en los ojos con lágrimas mirando sus solitarias manos". Y el dolor acumulado produce siempre pérdida de la esperanza. La Procuraduría para la Defensa de los Derechos Humanos, entre otras instituciones y personas, tiene que ser símbolo de esperanza en un El Salvador en el cual las institucio- nes y quienes las regentan han ido sufriendo un paulatino y severo desgaste. El deseo de que todos podamos vivir con dignidad y justicia, gobernados por leyes que regulen adecuadamente la convivencia ciudadana, y por instituciones que las apliquen sin doblegarse ante la voz del más fuerte, tiene que encontrar eco no solamente en personas o instituciones privadas, sino sobre todo en instituciones públicas y en dependencias del Estado. Al próximo procurador o procuradora le corresponde también la tarea de generar esperanza.

Aunque de esta situación no se sale con el apoyo de una sola institución, es evidente que la Procuraduría para la Defensa de los Derechos Humanos puede ser fermento para la recuperación de la confianza ciudadana en las instituciones. De hecho, históricamente, durante los tres años de la administración de la Dra. Victoria de Avilés, se convirtió en una luz de esperanza para muchas y muchos salvadoreños. Lo que en aquel momento se centró en la honestidad, valentía y voluntad clara de defender a los más débiles de la sociedad y sus derechos, debe continuarse en nuestros días. Pero debe también ampliarse a una serie de responsabilidades que tocan el bien común y que iremos mencionando.

En primer lugar, el procurador salvadoreño tiene como mandato explícito la defensa de los derechos humanos. En otros cargos semejantes, el mandato no es tan explícito, sino que se refiere a 
la defensa del ciudadano frente a la arbitrariedad, mal funcionamiento o abuso del poder público. Los derechos humanos ofrecen un marco mucho más amplio, puesto que éstos pueden ser violados tanto por el Estado como por los particulares. Por transnacionales o incluso por aquellos sectores de la sociedad civil que tienen de alguna manera monopolizadas funciones de servicio público que deben estar abiertas a todos los ciudadanos. En lo que respecta a funciones de interés público monopolizadas, podemos señalar al sector de los medios de comunicación, que evidentemente violarían derechos básicos (Artículo 19 de la Declaración Universal de Derechos Humanos) si impidieran la libre expresión de ideas a través del manejo arbitrario, exclusivista e irresponsable de sus dueños. El hecho de que los sistemas de protección de derechos humanos hayan sido diseñados como remedio, prácticamente exclusivo, para las violaciones por parte del Estado, hace más necesaria la presencia en nuestro país de una procuraduría identificada plenamente con la defensa de los derechos humanos. Porque, efectivamente, éstos son violados también por empresarios, por dueños de medios de comunicación, o por particulares. Esto, además, sucede con frecuencia, dado el desarrollo insuficiente de la legislación estatal en defensa de los derechos humanos, o la facilidad con la que se traspasan y olvidan recomendaciones de los organismos internacionales.

En este sentido, es importante que el procurador tenga una formación adecuada en legislación internacional y derechos humanos y dé garantías de su compromiso con los mismos. Pero más que a un conocedor exhaustivo del derecho, necesitamos a una persona convencida de la igual dignidad de la persona humana, dispuesta a defenderla ante cualquier tipo de agresión, discriminación o minusvaloración de la misma. Y capaz de utilizar sin miedo, de un modo adecuado y explícito, los diversos instrumentos que ofrece la Declaración Universal de los Derechos Humanos y los diversos protocolos y la doctrina posterior.

Esta tarea inicial y prioritaria se desglosa en muy diversas responsabilidades. Investigar, defender a las víctimas del mal funcionamiento de las instituciones estatales o de sectores privados que abusen de su poder, supervisar el funcionamiento de las instituciones de las que depende la seguridad o la protección de los derechos de las personas, revisar la legislación o promover la misma, encarar los problemas estructurales económicos, culturales y sociales que afectan negativamente o marginan y excluyen a la mayoría de la población del adecuado bienestar, etc., son funciones necesarias. Pero quisiera mencionar las que me parecen tareas prioritarias para el futuro inmediato.

(a) La supervisión de las funciones estatales y del respeto en ellas de los derechos humanos. En primer lugar, el procurador debe supervisar la actividad del Estado en todo lo que respecta a la relación con la persona y sus derechos. Viniendo de una cultura que aceptaba sin mayor crítica un Estado poco eficiente, en muchos aspectos con rasgos patrimoniales, y ciertamente autoritario, los abusos frente al ciudadano son frecuentes. Por ello, y porque el Estado es el instrumento básico de participación ciudadana desde donde se producen, o se deben producir, instrumentos adecuados para una pacífica y justa convivencia, la Procuraduría para la Defensa de los Derechos Humanos debe mantener una especial vigilancia del mismo. De hecho, son nuestros sistemas de gobierno y administración estatal, precisamente por la apropiación patrimonial del mismo, los que corren más riesgo de pervertir sus funciones de servicio, y de generar y encubrir situaciones de injusticia que claman al cielo.

Lograr que las instituciones funcionen, que la legislación vigente se cumpla, que las injerencias externas y las presiones, sean políticas, ideológicas o económicas, no impidan el adecuado y justo funcionamiento institucional, constituye una tarea de especial importancia derivada de esta labor supervisora. Los poderes fácticos, acostumbrados a funcionar al margen de la ley, o utilizándola a su capricho, frente a una débil institucionalidad estatal, constituyen una seria amenaza de perversión de la función pública. Erigirse en defensora de la institucionalidad y del funcionamiento apropiado de las leyes y de sus administradores es, en este contexto, tarea prioritaria.

(b) Ampliación de mecanismos de defensa de los derechos humanos y aplicación de los mismos a toda la sociedad. Pero este servir de instancia moral entre los diversos poderes del Estado y la persona, defendiendo a esta última frente a los posibles abusos, debe enmarcarlos la Procuraduría para la Defensa de los Derechos Humanos en un conocimiento adecuado de la realidad socioeconómica y cultural del país. En El Salvador, la pobrezá, en términos economicistas y reducidos a fórmula matemática, afecta a la mitad de la pobla- 
ción. Pero si saliendo de las fórmulas nos referimos a necesidades básicas de consumo, es probable que afecte al 70 por ciento de los salvadoreños. Esto se agrava, además, con una situación de desigualdad en el reparto de la riqueza que hace percibir al 20 por ciento más rico de la población un ingreso 16 veces superior al ingreso destinado al 20 por ciento más pobre. Esta situación carente de equidad, que se extiende después a dimensiones educativas, culturales y de salud, y que viene reforzada por políticas despreocupadas en la lucha contra la pobreza, hace que la mayoría de los salvadoreños no sólo puedan ver violados sus derechos por el Estado, sino por cualquier particular individuo o institución que acumule fuerza y poder. En ese sentido, sería absurdo que la Procuraduría para la Defensa de los Derechos Humanos cayera en el falso mito de que sólo el Estado viola los derechos humanos. Un empresario poderoso puede individualmente violar derechos humanos, así como otros entes u organizaciones políticas, gremiales o empresariales. En ese sentido, se vuelve urgente trabajar en la ampliación de los instrumentos de defensa del ciudadano frente al Estado, desarrollándolos y aplicándolos a otras fuentes institucionales o personales de violación de los derechos humanos. Para ello deben impulsarse mecanismos de mediación, incluso dentro de la sociedad civil, que permitan el respeto a los derechos humanos en las instituciones que por una u otra razón acumulan poder. Y si éstos fracasan, mecanismos claros de defensa de los derechos básicos.

(c) Educación en derechos humanos. En muchos estados, incluido El Salvador, los procesos de violación de los derechos de las personas suelen ir acompañados de pautas culturales de justificación del abuso. El ejemplo más patente puede ser el machismo, que durante tanto tiempo ha justificado la irresponsabilidad paterna en la familia y el maltrato de la mujer, con todas sus secuelas de exclusión, golpes; violaciones e irrespeto absoluto a valores e integridad femenina. De hecho, ya la Procuraduría para la Defensa de los Derechos Humanos responde a este tipo de problema social y cultural con la creación de adjuntías dedicadas a la protección de la mujer y la niñez, víctimas principales de este tipo de comportamiento cultural.

Más larvado, pero no menos grave, es el escaso valor que se le da a la vida humana. Algunos de los casos que ha tenido que enfrentar El Salvador en el pasado y que, en cierta manera, continúan en el presente, tienen que ver con el desinterés por las víctimas y por la tendencia a negociar con los fuertes y plegarse a sus presiones en casos donde el derecho a la vida ha sido violado. Los resultados en el caso de Katya Miranda o en el doble atropello de la Zona Rosa bajo efectos del alcohol, no nos dejan mentir. Como tampoco el caso de un diputado suplente que tras haber ultimado a dos personas en un autobús consiguió, sin que se llegara ni siquiera a discutir un antejuicio, que su caso no fuera remitido a los tribunales con la excusa de que nadie había reclamado en favor de los muertos. Esta cultura lleva a una valoración infame de las víctimas, cayendo con frecuencia en un tipo de conducta que no podría ser catalogado más que como racista, aunque no sea la raza, sino el estatus económico y social el que determine los derechos e incluso las medidas a tomar tras los homicidios. El envenenamiento, con clara responsabilidad criminal, de más de 125 alcohólicos, sin que al caso se le dé la debida importancia y seguimiento, muestra el rostro triste de una sociedad que minusvalora y pasa indiferente ante la muerte de los pobres y excluidos de nuestra sociedad. Defender la vida con claridad, especialmente cuando los atentados contra la misma afecten a personas o grupos sin defensa y provengan de estratos acostumbrados a la impunidad, es un imperativo absoluto, tanto para la Procuraduría para la Defensá de los Derechos Humanos como para todos los interesados en que los derechos fundamentales de la persona se respeten.

Los ejemplos puestos nos hablan de la importancia de desarrollar una labor educativa en derechos humanos. $Y$ aunque la misma es imposible que sea realizada exclusivamente desde la Procuraduría, sí es importante que esta institución abandere un auténtico movimiento que incluya a sectores estatales y particulares en la promoción, educación y formación en derechos humanos. 
(d) Defensa de los derechos económicos y sociales. La situación salvadoreña hace indispensable también que la Procuraduría para la Defensa de los Derechos Humanos mantenga una defensa adecuada de los derechos que habitualmente llamamos económicos y sociales. La erradicación de la pobreza no puede contemplarse como una meta exclusiva de los economistas y de instituciones especializadas. Al ser la misma, uno de los obstáculos principales para la vigencia plena de los derechos humanos, la Procuraduría debe mantener una especial vigilancia al respecto. Ello implica una relación muy cercana de ella con entes especializados, y un análisis permanente de todas aquellas estructuras jurídicas, sociales o económicas que puedan consagrar estados de exclusión o fomentar directamente la pobreza. El tema impositivo, como forma de redistribución de la riqueza, no tanto en sus dimensiones teóricas, sino en su ejecución práctica, debía recibir una especial atención. Como también la organización de servicios que respondan a derechos básicos de las personas. $\mathrm{Y}$ a este respecto, valga poner como ejemplo de la estructuración estatal injusta y violatoria de la igualdad en el terreno de los derechos al servicio público de la salud.

El derecho a la salud es básico para la felicidad individual, imprescindible para el desarrollo económico e independiente de la condición social de la persona. Sin embargo, en El Salvador, el Estado organiza la respuesta a este derecho de todos los salvadoreños en función de la capacidad de cotizar de las personas. El económicamente pobre goza, en este aspecto, de una menor protección de su derecho. $\mathrm{Y}$ así, el doble sistema de salud pública atiende a una cuarta parte de la población desde el seguro social, con prestaciones de relativa calidad, mientras margina al 75 por ciento de la población a una atención en el sistema del Ministerio de Salud de muy inferior calidad. Algo semejante podríamos decir de las pensiones de jubilación y de otros derechos básicos, como la educación, en donde la capacidad de cotización da una oportunidad superior y margina casi absolutamente a una alta proporción de la población. Vigilar la estructuración oficial de servicios, sobre todo si se convierte en violatoria de la igualdad de oportunidades y derechos, constituye una especial responsabilidad, más allá de la denuncia de violaciones particulares.

Los derechos que se suelen llamar de tercera generación deberían también ser parte de este mis- mo esfuerzo. La defensa del medio ambiente en un país como el nuestro, en el que se dan círculos infemales de degradación ecológica, que combinan muy diversos elementos para producir más pobreza e impedir el acceso al desarrollo, no podemos ni debemos olvidarlo desde la defensa de los derechos humanos.

(e) Vigilancia y promoción de una legislación respetuosa con los derechos humanos. Este conglomerado de diversas problemáticas, tan imbricadas unas con otras, y tan derivadas de una situación de injusticia estructural, debe llevar también a la Procuraduría para la Defensa de los Derechos Humanos a vigilar especialmente la legislación vigente y la producción de nuevas leyes. $E$ incluso en ocasiones a iniciar y promover determinados puntos de legislación o a interponer los recursos pertinentes, incluso de amparo, frente a leyes que amenacen la perpetuación de situaciones violatorias de los derechos humanos.

Esta responsabilidad abarcaría un doble aspecto. El primero de ellos consiste en defender el cumplimiento, e incluso promoverlo, de la legislación vigente. Para nadie es un secreto que determinados puntos constitucionales no se cumplen en el país. El Ordinal 12 del Artículo 38 de la Constitución, que garantiza el derecho a la indemnización universal, duerme en el sueño, no precisamente de los justos, desde 1983, por ausencia de regulación en la ley secundaria. Artículos como el 65, 69 y 70, referidos al campo de la salud no son cumplidos ni de cerca por el Estado. El derecho de respuesta, garantizado en el Artículo 6 de la Constitución, no sólo no se respeta en el país, sino que incluso algunos de los grandes medios se dan el lujo de defender públicamente posiciones en clara contravención del mismo. Siendo la Constitución el marco básico de justa y pacífica convivencia, la Procuraduría para la Defensa de los Derechos Humanos debería dedicarle una especial atención, tanto en los casos en que se viola el espíritu de la misma como cuando instituciones públicas o privadas la ignoran y desprecian, especialmente en lo que toca a derechos fundamentales o a aspectos concretos de la promoción y cuidado de los mismos.

Además de la defensa de los derechos no respetados, a pesar de estar explicitados y protegidos en la Constitución, la Procuraduría para la Defensa de los Derechos Humanos debería vigilar con atención la aparición de leyes que tienen que ver con la convivencia y los derechos ciudadanos. 
Con cierta frecuencia aparecen leyes lesivas para el bien común, las cuales terminan dañando aspectos fundamentales de los derechos humanos. Frente a ellas debe haber una posición reflexiva, educativa y activa, cuando no de denuncia o de amparo contra la misma. Un ejemplo interesante podría ser la nueva ley de armas, más centrada en los intereses de los vendedores de la mismas que en la seguridad ciudadana. El simple hecho de que esta ley otorgue más facilidades al Procurador para la Defensa de los Derechos Humanos para portar armas, que al resto de los ciudadanos, debería haber motivado una intervención pública inmediata del titular del cargo, protestando por el contenido simbólico de una legislación que facilita la tenencia de armas a quienes detentan puestos que deben servir especialmente a la pacificación del país y no a la proliferación de signos de violencia. La ley del impuesto al valor agregado o cualquier otra ley susceptible de dañar de un modo injusto y poco equitativo a los más pobres del país debería ser analizada en detalle, así como vistas en su conjunto. Pues, incluso, una ley de impuestos regresiva, si se aplica en el contexto de una política en que la mayoría de lo recaudado retorna en forma de servicios a los ciudadanos más pobres, puede contribuir al final al bien común.

Dentro de este contexto, la Procuraduría para la Defensa de los Derechos Humanos podría ejercer también su derecho a promover reformas legales, impulsar nueva legislación de defensa de la persona (por ejemplo, la unificación de los sistemas de salud, la ampliación de pensiones a los trabajadores del campo, la universalización del décimo tercer mes, etc.), o ejercer el amparo constitucional contra normas que afecten seriamente los derechos de las personas.

(f) Colaboración con la sociedad civil. Finalmente, es importante reseñar la importancia de la cooperación con todos aquellos grupos, organizaciones no gubernamentales, sectores de la sociedad civil que tienen como punto importante de sus agendas el tema de los derechos humanos. La procuraduría encargada de su defensa no puede trabajar sola, sino que debe incluir a todas aquellas personas que de alguna manera se sienten comprometidas con la igual dignidad de la persona humana y el respeto, plasmado en derechos, que ésta se merece, En primer lugar, el trabajo activo en colaboraciớn con las organizaciones no gubernamentales específicas que se mueven en el campo de los derechos humanos es importante. En este campo, nadie tiene el monopolio, porque todos nos movemos en un ámbito de interés, propiedad y herencia común. El mutuo apoyo en formación, en investigación, en aprovechamiento de experiencias, en diálogo, e incluso en debate crítico, se tornan fundamentales para el desarrollo de la tarea que se quiere llevar adelante.

Esta colaboración, además, no debe limitarse a la sola relación con las organizaciones no gubernamentales defensoras de los derechos humanos. Ampliar la relación hacia lo que llamaríamos el mundo interdisciplinar de la democracia sería indispensable para obtener éxito en algunas de las tareas pendientes y esbozadas anteriormente. Los economistas, los expertos en tecnología aplicada, especialmente en el campo de la ingeniería, los conocedores de la problemática de la salud pública y preventiva, el movimiento ecologista, los que reflexionan sobre el mundo de los valores y sus aplicaciones prácticas, las iglesias y un largo etcétera constituyen la amplia y necesaria comunidad que puede dar vida y eficacia a estos esfuerzos en favor de los derechos humanos. Invitar, incluir, incorporar, animar al compromiso con los derechos humanos a sectores en ocasiones olvidados, pero indispensables para la realización social de los mismos, son palabras a las que tendremos que acostumbrarnos en el futuro para tener éxito en lo que pretendemos.

De las líneas brevemente esbozadas se puede deducir con facilidad que nos movemos en un campo no conocido ni apreciado de formas adecuadas. Las declaraciones oficiales de algunos partidos en favor de los derechos humanos están con frecuencia teñidas de demagogia, de oportunismo y de desinterés real. Algunos empresarios no dudan en afirmar que los derechos humanos son parciales, instrumento político de la izquierda y demás parlanchinería semifascistoide de la derecha ignorante tan típica en nuestro medio. No nos encontramos, pues, con un camino que podamos calificar como sencillo y fácil. Al contrario, o lo recorremos juntos o la amenaza de marcha atrás pronto dejará de ser un peligro para convertirse en una triste realidad.

Es evidente que queda mucho por decir de la Procuraduría para la Defensa de los Derechos $\mathrm{Hu}$ manos y del perfil que la misma debería tener en los próximos años. Pero estoy seguro de que si los puntos señalados se trabajan en conjunto entre esta institución, las organizaciones no gubernamentales 
y la sociedad civil, la propia realidad hará que muchos de los puntos no tratados salgan a la luz, se trabajen pluridisciplinarmente y se logre la finalidad que todos pretendemos: una sociedad donde la mejor herencia del siglo XX, la convicción de la igual dignidad de la persona, encuentre su plenitud en el nuevo siglo a través de la construcción solidaria y libre de una nueva sociedad.

José M. Tojeira S. J. Rector de la Universidad Centroamericana "José Simeón Cañas" 